\title{
Recent Developments and Potential Challenges in Dealing with Internal and External Surfaces of Glass Matrix and Resin Matrix Ceramic Materials. A Literature Review
}

\author{
Alaa Samir El-Ashkar* and Omnia Nabil \\ Department of Fixed Prosthodontics, Faculty of Dentistry, Cairo University, Egypt \\ *Corresponding Author: Alaa Samir El-Ashkar, Department of Fixed \\ Prosthodontics, Faculty of Dentistry, Cairo University, Egypt.
}

Received: January 13, 2022

Published: February 24, 2022

(C) All rights are reserved by Alaa Samir

El-Ashkar and Omnia Nabil.

\begin{abstract}
Objectives: Glass matrix and resin matrix ceramic materials has gained popularity in recent years, which has increased their clinical use. The purpose of this scientific overview is to discuss the impact of existing internal and external surface treatment procedures, as well as their potential consequences.

Study Selection, Data and Sources: The recent literature of in vitro research investigating adhesion methods and external surface techniques on both glass matrix and resin matrix ceramics, including studies from 2014 until 2020, was considered. A search of English language studies was carried out using Pubmed, MEDLINE, and Google scholar.

Results: A total of ninety-seven publications were recognized for evaluation. Several surface treatment techniques with different testing approaches were reviewed and categorized in this review according to the surface modification method: internal surface treatments including mechanical bonding, chemical etching, and external surface treatments including glazing and polishing.

Conclusions: With respect to the diversity of the included studies, their results were difficult to compare in that the factors varied in each research protocol. This was considered a limitation for this review to recommend a final surface treatment protocol for each material. However, most of the studies reported hydrofluoric acid (HF) as the most effective in glass matrix ceramics internal surface treatment. Increasing the concentration and etching duration maximizes the etching effect. For resin matrix ceramics, air particle abrasion and hydrofluoric acid etching have been widely accepted as micro-mechanical internal surface treatment protocols. The merits of various glazing and polishing surface treatment systems for the two ceramic materials were also debated in other studies. Depending on the ceramic microstructure, exterior surface investigations favoured one approach over the other. A final recommended protocol, however, has not yet been authorized.
\end{abstract}

Keywords: Glass Matrix Ceramics; Resin Matrix Ceramics Internal Surface Treatment; Polishing; Glazing; Bonding; Silanization; Neutralizing Agents; Cleansing Agents; And Laser Surface Treatment

\section{Introduction}

Ceramic surface treatment performance is crucial for a strong and long-standing resin cement bond. Two fundamental methodologies have been established for ceramic restoration: micromechanical retention and chemical bonding. Glass matrix ceramics are rich in silica phases in which hydrofluoric acid selectively attacks, changes the surface topography, and creates microporosities that enable micromechanical interlocking of the resin cement. However, hydrofluoric acid substitutes were introduced as an alternate solution to the late acid drawbacks. Silane coupling agents 
are intermediaries. They enhance chemical bonding of the resin cement with the etched ceramic surface with limited data on new monomers as substitutes. Glazing has been the gold standard for external surface treatments for glass matrix, with few drawbacks. Rupture of the glazing layer increases the roughness of the surface, resulting in clinical concerns such as opposing tooth wear, microbial biofilm retention, periodontal tissue irritation, staining, and lower crack propagation resistance. The researchers advocate reglazing or polishing ceramic restorations as options to attain improved surface smoothness for the reasons stated above. Resin matrix ceramics are a relatively new addition to the dental ceramics classification. Scientists are investigating the bonding and finishing methods for these materials in an effort to determine which is the most dependable. The aim of this review was to evaluate invitro studies investigating various surface treatment protocols for both internal and external surfaces of glass matrix and resin matrix ceramics.

\section{Materials and Methods}

\section{Search Strategy}

The available data was reviewed using the Google Scholar and PubMed databases. On March 15, 2020, the study was performed. The review covered papers that were published between January 1, 2014, and January 1, 2020. The research papers included in this review studied the multiple approaches for treating the internal and external surfaces of zirconia restorations. Invitro investigations, reviews of literature, and systematic reviews are the focus of the research. The subsequent search terms, as well as their combinations, were investigated: "Glass matrix ceramics", "Resin matrix ceramics", "Internal surface treatment”, "Polishing”, "Glazing”, "Bonding", "Silanization", "Neutralizing agents", "cleansing agents", and "Laser surface treatment" (Table 1).

\begin{tabular}{|l|c|}
\hline Topics & Criteria \\
\hline Data base & Google scholar, PubMed, MEDLINE \\
\hline Date range & 2014-2020 \\
\hline Keywords & $\begin{array}{c}\text { Glass matrix ceramics, Resin matrix ceramics } \\
\text { Internal surface treatment, Polishing, Glazing, } \\
\text { Bonding, Salinization, neutralizing agents, } \\
\text { cleansing agents, and Laser surface treatment. }\end{array}$ \\
\hline Language & English \\
\hline Type of paper & $\begin{array}{c}\text { Invitro studies, Review of literature, system- } \\
\text { atic review }\end{array}$ \\
\hline Type of journal & Dental Journals \\
\hline
\end{tabular}

Table 1: Criteria for research selection for this review.

\section{Articles reviewed and data extraction}

Two evaluators read each of the included articles independently. Invitro studies evaluating various internal surface modification and priming techniques for glass matrix ceramics and resin matrix ceramics were used as inclusion criteria. Furthermore, investigations examined various exterior surface techniques in terms of polishing, glazing, and coating. Exclusion criteria were studies on: (1) Zirconia; (2) Intra-radicular posts; (3) Implant superstructure; (4) Polycrystalline ceramics.

\section{Results}

A total of 97 articles were selected after searching and scanning the databases. It was impossible to examine the data quantitatively due to the significant variety of research publications in terms of study design, testing methodologies, and outcome factors. In terms of surface treatment approaches, the articles are summarized in figure 1.

\section{Discussion}

\section{Glass matrix ceramics}

Internal surface treatment methods of glass matrix ceramics

Various internal surface treatment methods are mentioned in the literature for glass matrix ceramics. These methods are divided into chemical conditioning with hydrofluoric acid, other acids, and mechanical conditioning such as sand blasting and lasers. Yet the chemical conditioning method is the most commonly used.

Chemical surface treatment methods of glass matrix ceramics The hydrofluoric acid

HF chemical conditioning of glass matrix ceramics changes the surface topography (by selectively dissolving the glassy phase and forming surface hydroxyl groups $(-\mathrm{OH}))$. The micromechanical surface changes are primarily caused by the dissolution of the glassy phase in the glass produced by the fluoride substitution to oxygen in the Si. F4 glass. HF acid is a weak acid. Thus, the glass matrix dissolution process does not rely on the "acidic" property of HF. Basically, the term "Acid Etch" is a misrepresentative term and, therefore, "HF etching" is favoured. Despite the fact that HF is considered a golden standard for chemical conditioning, the use of HF acid is hazardous and encounters some drawbacks. When dissolved, the fluoride ion is released and can penetrate tissue, leading to skin burns and various systematic problems because of its high toxicity [1]. The risk of over-etching, H-F residual acid might be entrapped inside the crevices, or the surface irregularities will continue to 
dissolve the surface, which will adversely affect the bond strength. Moreover, the acidic medium of the H-F residues affects the resin polymerization and thus results in poor adhesion strength or poor bond strength [2]. correspondingly, the concentration over etching, $10 \%$ HF demonstrated the most evident etching pattern with unsupported crystals and areas with topography similar to over-etching. While the partially supported crystals contained by the glassy matrix were absent, with an etching time extended to 20 seconds. This adversely affected the lower flexural strength of the specimen. Even with the buffered HF, the same over etching pattern was noted [3]. Preheating has the potential to improve the bond strength of lithium disilicate glass ceramic with a $5 \% \mathrm{HF}$ concentration. Sundfeld., et al. "found that preheating $5 \% \mathrm{HF}$ at $70^{\circ} \mathrm{C}$ increased micro shear bond strength values for $5 \% \mathrm{HF}$, with statistically similar re- sults to non-preheated $10 \% \mathrm{HF}$ at room temperature. While preheating, both the ceramic surface and the HF showed no statistical significance between other groups. This can be attributed to heat acting as a catalyst by dramatically increasing the rate of chemical reactions as temperature rises. The heat treatment causes the ionised HF to become more agitated, causing it to move faster and cause more vigorous collisions with lithium disilicate, resulting in greater removal of glassy matrix. When compared to $10 \% \mathrm{HF}$ at room temperature, the preheated $5 \% \mathrm{HF}$ achieves statistically similar micro shear bond strength values and etching patterns [4]. A number of chemicals were introduced to substitute HF. Some H-F Substitutions were point out in literature as Phosphoric acid, acidulated phosphate fluoride (APF), Ammonium bifluoride (ABF), Ammonium polyfluoride, Buffered Hydrofluoric acid.

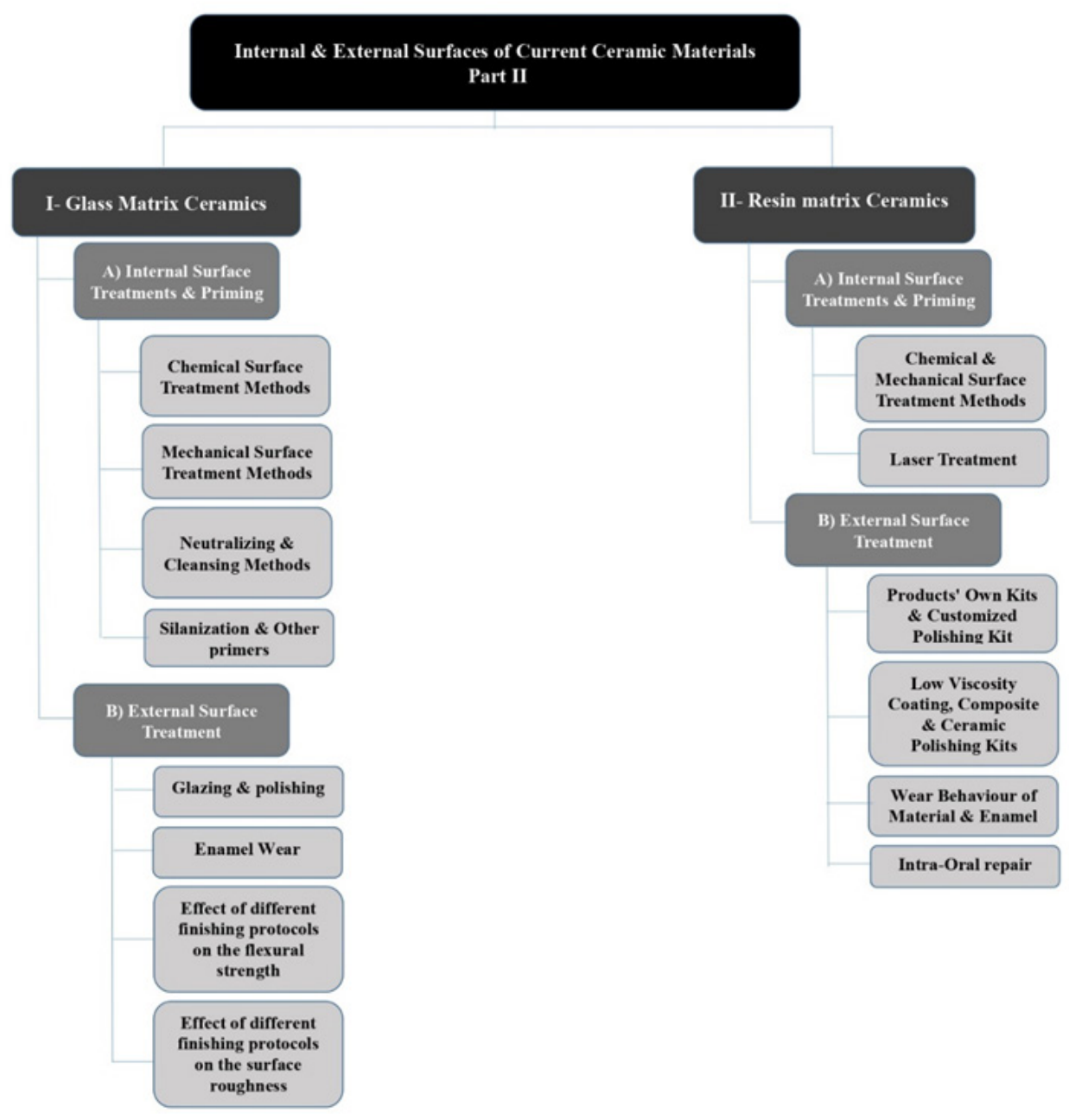

Figure 1: Illustrates a schematic description of the surface treatments discussed in this review for both glass matrix ceramics and resin matrix ceramics. 


\section{HF acid substitutes}

\section{Phosphoric acid}

The application of $40 \%$ phosphoric acid for $5 \mathrm{~s}$ or $60 \mathrm{~s}$ did not show any obvious morphological change on the ceramic surface under SEM observation. This might be attributed to the acidity being inconsequential in the etching process, whereas the title role of fluoride in the atomic displacement with silicon dioxide is of major significance [1]. In conclusion, 37.5-40\% phosphoric acid makes a good cleanser for the porcelain surface and fluoro-silicate deposits [2].

\section{Acidulated phosphate flouride}

When the etching time with $1.23 \%$ acidulated phosphate fluoride (APF) gel of the leucite-reinforced ceramic was extended up to 10 minutes, the resultant shear bond strength value was insignificantly divergent to that after etching with $9.6 \% \mathrm{HF}$ acid for 4 minutes. The APF is mostly efficient with leucite-based ceramics rather than lithium disilicate, because the dissolving behaviour and pattern differ between leucite and lithium disilicate containing glass ceramics. The crystalline leucite has a faster dissolution rate than lithium disilicate crystals [5].

\section{Ammonium Bi-fluoride (ABF)}

$\mathrm{ABF}$ is less toxic and hazardous than $\mathrm{HF}$. ABF forms a linear defect etching pattern because it mainly attacks phase boundaries and cracks that already exist or are caused by leucite crystals in leucite-reinforced glass matrix ceramics. Carpena., et al. "reported that the etching patterns of ABF are very similar to those created when HF is applied for a shorter time and at a lower concentration. Carpena., et al. "concluded that etching current glass matrix ceramics with HF results in statistically higher mean bond strengths compared to etching with $\mathrm{ABF}[3]$.

\section{Ammonium poly-fluoride (APF)}

APF is a glass matrix etchant, notably less toxic than HF. In selfetching ceramic primer (Monobond Etch and Prime, Ivoclar Vivadent, Schaan, Liechtenstein), APF stabilises the silane coupling agent in a highly reactive form during storage. Volkel., et al. "the mono-bond etch and primer etching effect was less evident than the etching effect of HF acid, yet adequate to produce sufficient micro-retention for a reliable adhesive bond. The Mono-bond etch and primer (Ivoclar Vivadent) did not cause over-etching to the glass matrix ceramics surface when the etching time was doubled.
Unlike 5\% HF, which causes over etching and detached lithium disilicate crystals [6].

\section{Buffered oxide etch (BOE)}

Also known as buffered HF or BHF, Its primary use is in the etching of silicon dioxide (SiO2) [6]. It is a mixture of a buffering agent, such as ammonium fluoride (NH4F), and hydrofluoric acid (HF). Antonio., et al. "investigated the etching efficacy of both $5 \%$ HF and 9\% Buffered H-F. The findings showed that the HF 5\% displayed higher shear bond strength analysis at 15 seconds. While 9\% HF recorded a lengthened reaction time because of its buffered composition. The author recommended HF etching with concentrations of $5 \%$ or $9 \%$ for $15 \mathrm{~s}$ to $60 \mathrm{~s}$ as a surface pre-treatment of Vita Mark (VITABLOCS ${ }^{\circ}$ from VITA Zahnfabrik), VITA Enamic from VITA, e.max (IPS e.max CAD, Ivoclar Vivadent) and VITA Suprinity", (VITA Zahnfabrik) [7].

Mechanical surface treatment methods of glass matrix ceramics

Sand blasting or air particle abrasion (APA) is done to roughen the ceramic surface by blasting it with alumina (Al203) particles. 25 to 50 um alumina powder is ordinarily used in glass ceramics at a pressure of $0.28 \mathrm{Mpa}$ [1]. The negative aspect of the sandblasting method is the formation of surface micro-crack in the course of the impact of the alumina powder particles on the glass matrix ceramic. This might adversely affect the mechanical strength of the surface layer, which would basically affect its durability and clinical performance.

Uwalaka., et al. "evaluated the influence of APA on the flexural strength of glass matrix ceramics. The sandblasting caused an array of critical defects, which under tensile stress instigate early failure in innumerable failure manners, originating at occlusal contacts or cementation surfaces. Fracto-graphic analysis of the failed bi-axial flexural strength samples showed fracture origins. In the lithium disilicate specimens, the fracture initiated at the resin-ceramic interface, while the leucite samples demonstrated interfacial and interior flaw failure [8]. Sandblasting (Al203, 30 m, 1 bar) of zirconia-reinforced lithium-silicate ceramic (VITA Suprinity", VITA Zahnfabrik) has also been shown to significantly reduce flexural strength [9].

Concerning bonding, the air particle abrasion might contaminate the surface with alumina powder particles. After salinization, 
Al-O-Si linkages can be formed on the alumina particle contaminated surface. These show weaker bonding links than-Si-O-Si-and are more susceptible to bond degradation [10].

In recent literature, lasers have also been used to alter the glass matrix ceramic surface. The laser can eliminate particles by means of an ablation process, which consists of micro-explosions and vaporization. The surface is exposed to laser beam radiation. The laser energy is absorbed and transformed into heat energy. The heat energy leads to melting of the surface and creates surface irregularities. The resultant surface modifications enhance adhesion. Nd: YAG and Er: YAG lasers were evaluated in terms of surface roughness and bond strength of glass ceramics [11,12].

For the Feldspathic ceramics, the surface was randomly melted and corroded without any fissures or cracks in both lasers [11]. In Liu., et al. "study, the Nd: YAG laser was shown to be as effective as HF for etching the surface of feldspathic porcelain [12]. In contrast to Akpinar., et al. "the Nd: YAG laser achieved lower bond strength compared with HF and sandblast. While the femtosecond laser could create stronger bond strength [13], SEM observation revealed that the Er: YAG laser created irregular lithium disilicate crystals on the surface of the lithium disilicate glass matrix ceramics. The laser power was directly proportional to the surface irregularities. The higher the laser power, the greater the irregularities of the ceramic surface $[1,11]$.

\section{Neutralizing and cleansing methods}

Some HF acid remnants might be left inside the crevices of the etched surface. These residues lower the $\mathrm{pH}$ value of the etched ceramic surface, which undesirably affects the bonding by hindering the polymerization of resin cement. Also, low $\mathrm{pH}$ fastens the hydrolysis reaction of silane, affecting the bond strength and stability [14].

Succeeding surface treatment and conditioning sequential steps of glass matrix ceramics, a neutralizing step is essential to reactivate the HF etched ceramic surface and remove the acid remnants [9]. The aim of neutralizing agents is to counterbalance the $\mathrm{pH}$ of the etched ceramic surface, correspondingly arresting any further topographical changes by the HF action. Thus, they significantly improve the bond strength with resin cements.

\section{Neutralizing agent}

Their mechanism of action mainly relies on calcium, which is the most common ingredient in neutralising agents. Calcium gluconate is chiefly used to cure HF burns. Calcium ion binds to fluorine ion on the etched ceramic, causing it to rinse away, leaving a clean, reactive, high surface energy surface for silane to bond to. The neutralising agent promotes the bond strength and does not have a direct effect on bonding. Ploypim., et al. "evaluate the effect of neutralising agents on the shear bond strength of hydrofluoric HF etched porcelain in non-aging and aging conditions. The results showed that the calcium gluconate and calcium carbonate groups reported the highest values comparable to the HF and ultrasonic groups. The application of neutralizing agents did not affect the bond strength in either the non-aging or aging conditions [14].

\section{Cleansing agents}

Saliva contamination of the restoration during the try-in procedure might form a protein layer on its surface. Such contamination could undesirably affect the bonding of restorations and subsequently lead to loss of the restoration. The aim of cleansing agents is to remove Fluorosilicate deposits resultant from the H-F etching, salivary proteins contaminating try in paste remnants after try in procedure and any other contaminants on the etched surface [15]. From the cleansing agents used in literature: a) Water spray and air drying. b) Sodium hypochlorite, c) Air polishing device with sodium bicarbonate, d) 37\% phosphoric acid, e) 96\% isopropanol and f) Re-etching with 5\% hydrofluoric acid [15]. The universal cleaning paste, Ivoclean (Ivoclar Vivadent), is reported as a valuable agent for the decontamination of ceramics. It has proven its efficiency in cleaning saliva contamination on zirconia ceramics. Despite this fact, its effect on the bond strength of lithium disilicate is not entirely clear. Comlekoglu., et al. "evaluated three cleaning methods (water spray, sodium hypochlorite, and cleaning paste) after saliva contamination and reported that all the methods did not increase bond strength [15]. Yoshida., et al. "water rinsing of saliva-contaminated lithium disilicate resulted in lower surface energy of substrate than non-contaminated lithium disilicate [16].

Dos Santos., et al. "evaluated the effect of 37\% phosphoric acid and ultrasonic cleaning used to remove the remaining hydrofluoric acid on the shear bond strength (SBS) between lithium disilicate and resin cement. The ultrasonic cleaning showed complete removal of all surface contaminants (fluorosilicate deposits and salivary proteins). The phosphoric acid didn't efficiently remove the contaminants [17] Kim., et al. "ultrasonic cleaning was more effective in contaminant removal than air-water spray cleaning. It is 
due to the fact that the effect of high-frequency vibrations results in the detachment of salivary contaminants from the lithium disilicate surface [18]. In accordance with Sriamporn., et al. "who reported that ultrasonic cleaning after etching with HF for 5-10 minutes in distilled water resulted in maximum cleansing and optimal bond strength [14] Lapinska., et al. "reported that HF acid re-etching after saliva treatment was the most effective method for removing lithium duplicates contaminated with saliva and/or a silicone disclosing medium [19]. In conclusion, ultrasonic cleaning removed fluorosilicate salts, neutralizing agent remnants, and H-F acid remnants. It also promoted the surface energy without changing surface topography.

\section{Silanization and other primers}

Conditioning the HF etched ceramic surface with a Silane coupling agent leads to the formation of covalent and hydrogen bonds, which improves the bond strength of the glass matrix ceramics. The silanes are bi-functional/dual reactive with an inorganic group that reacts with $\mathrm{Si}-\mathrm{OH}$ on the ceramic surface via siloxane bond and condensation reaction. They also have an organic group that can chemically bond to carbon double bond methacrylate-based resins. The most commonly used silane agent in dentistry is $\gamma$-MPTS or MTS, MPS, 3-methacryloxypropyltrimethoxysilane or $\gamma$-methacryl oxypropyltrimethoxysilane) [10]. Other silane agents proved their creditability, Tian., et al. "MPS is found to enhance the shear bond strength of the resin-ceramic compared to 3-methacryloxypropylmethyldimethoxysilane (MDS) [1].

The available silane liquid primers can be divided into, singleliquid silane primers (pre-hydrolyzed) or two-bottle liquid silane primers [1]. The efficiency of pre-hydrolyzed, single-bottle silane primers is unpredictable. Pre-activated silane primers may become turbid over time after the first use. This is due to the excess formation of siloxane oligomers/polymers that are inactive and effectiveness progressively decays over time. On the other hand, two-bottle solutions are preferred over one bottle. The two-bottle silane system has prolonged the shelf life and has higher initial reactivity [20]. It is advised to apply additional silane primer for optimal bonding to lithium disilicate restorations. Moro., et al. "recommended an additional silane application resulted in a higher $\mu$ SBS. Clinically, the use of a separate freshly mixed silane primer with the adhesive remains recommended to bond glass-rich ceramics [21]. Kim., et al. "found that silane treatment before prosthetic try in and saliva contamination resulted in a significantly higher bond strength than that after saliva contamination, regardless of the cleaning methods. This could be attributed to the hydrophobicity of the surface by silane treatment, and as a result, reduces the risk of saliva proteins adhering to the ceramic surface, which alters the bond formation between the silane and ceramic [18].

However, silanes have some drawbacks, like bond degradation and aging. It is also difficult to achieve a thin uniform monolayer of applied silane on glass matrix ceramics. Successive applications and multi-layers result in cohesive failure. Thus, a thin silane coat is recommended for achieving a durable bond. The layer applied should be a monolayer (1 layer) 10-50 nm thick). But the question remains how to control the thickness of the application. Silano pen can be one of the methods of controlling the thickness of the silane layer. A modification of the Silicoater ${ }^{\star}$ technique was familiarized successively as Silano Pen ${ }^{\circ}$ or PyroSil Pen (Bredent, Senden) for extra-oral use in dental laboratories. It is a hand-held device with a flame, heat treatment. The surface is heated with the "pale-blue" flame zone of the pen. The flame is continuously moved forward and backward across the surface to be treated so that uniform treatment of the entire surface is reached. After the heated areas cool down below $50^{\circ} \mathrm{C}$, the bonding agent is placed instantly. 10 Reports on this approach are few.

Applying the silane coupling agent with heat at $100 \mathrm{C}$ for $1 \mathrm{~min}$ ute via blow dryer or oven helps in: eliminating alcohol, water, and other by-products from the surface of the ceramic. The heat treatment helps with the accomplishment of the silane-ceramic condensation reaction, making the covalent bond more effective and resistant to aging/degradation. Andrea., et al. "used a silane coupling agent for 1 minute before drying with a warm air stream $\left(100^{\circ} \mathrm{C}\right)$. The authors used a miniature custom-made blow dryer delivering a constant flow of warm air, approximately $100{ }^{\circ} \mathrm{C}$ at $1 \mathrm{~cm}$ from the nozzle. The results showed that the warm air step achieved a significantly stronger adhesion than control groups [22]. Yavuz., et al. "studied the micro-tensile heat treatment of silane at different temperatures. The results showed that silane heated at $100{ }^{\circ} \mathrm{C}$ achieved higher values than at $60^{\circ} \mathrm{C}$. The least value was recorded by no heat treatment [22]. Abduljabbar., et al. "stated that the highest micro tensile bond strength values were achieved for lithium disilicate ceramics with heat-dried silane for 5 minutes at $100^{\circ} \mathrm{C}$ in a hot air oven. Heat treatment at $100^{\circ} \mathrm{C}$ has been shown to merge the layered surface, removing the interphase and increasing the bond strength of composite to ceramic [23]. De Carvalho., et al.

Citation: Alaa Samir El-Ashkar and Omnia Nabil. "Recent Developments and Potential Challenges in Dealing with Internal and External Surfaces of Glass Matrix and Resin Matrix Ceramic Materials. A Literature Review". Acta Scientific Dental Sciences 6.3 (2022): 61-72. 
"concluded that the heat treatment of the pre-hydrolyzed silane in an oven at $100^{\circ} \mathrm{C}$ for $2 \mathrm{~min}$ or with hot air application at $50 \pm 5 \stackrel{\circ}{\circ}$ for $1 \mathrm{~min}$, was effective in increasing the bond strength values between the ceramic and resin cement [24].

\section{Other ceramic primers}

Silane coupling agents are still the gold standard for adhesion promoters in dentistry. However, the bond degradation over time in the oral cavity is still a major concern. Recently, some approaches were tested to improve the bond durability, such as Silane molecule modification (synthesis) and the development of other coupling agents (such as multifunctional blends) [9].

Maruo., et al. "used a new silane agent of 8-MOTS (8-methacryloxyoctyl trimethoxy silane) molecule instead of conventional MTPS ( $\gamma$-methacryloxypropyl trimethoxy silane) in the pre-treatment of lithium disilicate. The pre-treatment with 8-MOTS silane increased the initial bond strength between lithium disilicate glass ceramic and resin. This could be attributed to the fact that the 8-MOTS has a longer hydrocarbon chain than $\gamma$-MPTS. However, the bonding performance of 8-MOTS did not differ from that of $\gamma$-MPTS. This study did not investigate the durability of bond strength achieved with 8-MOTS [25].

A blended silane system has been shown to enhance shear bond strengths between resin cement and ceramics. Blended silanes of 1,2-bis (trimethoxysilyl) ethane (BTS) and MPS could significantly increase the shear bond strength after thermocycling [10].

For the Zirconia-reinforced lithium silicate, 10-methacryloyloxydecyl dihydrogen phosphate (MDP) containing cement is recommended to be used after silanization of the HF etched surface. This can be attributed to MDP reacting with zirconia, forming a chemical reaction at the interfacial level between the hydroxyl groups present as a passive coating of zirconium oxide on the ceramic surface and the phosphate ester monomers of the MDP. Also increasing fracture resistance [26].

\section{Different external surface treatments modalities and compar-} isons

Ceramic restorations must have smooth surfaces to yield optimum esthetics, biological and mechanical properties. Rough restoration surfaces enable staining and allow plaque accumulation and can also cause abrasion and wear of antagonistic teeth. To minimize these risks, the ceramic surfaces must be smoothened as much as possible, accomplished by polishing and glazing.

\section{Glazing and polishing methods}

There are two kinds of glazing: auto glazing and over glazing. In the auto glaze, porcelain glazes itself by forming a surface layer containing a glass phase. For 1-2 minutes, rapidly raise the temperature to the fusion temperature in order to melt the surface particles. Colorless add-on glazing low fusing glass is available as a spray, powder, or paste that can be painted or sprayed onto a previously fired crown surface and fired again. On the milled glass matrix ceramic surface, pigments, characterizing agents, and over glaze can be applied using the "Combined Firing Process." The combined firing processing is extremely efficient and produces a highly aesthetic result quickly and easily. Laser glazing has recently gained popularity because the energy provided by the glazing treatments favors viscous glass flow inside irregularities, eliminating the need for an additional firing step in traditional glazing techniques. Polishing before glazing is highly recommended because it improves the final surface outcome in terms of light reflectance due to better glaze spread and reduces surface defects. After intra-oral occlusal adjustments, some authors preferred polishing over re-glazing. The polishing efficiency is determined by the ceramic's microstructure, the size of the polishing paste particle, the duration of the polishing procedure, and the number of steps in the polishing protocol. Each of the aforementioned external surface modality has an effect on the material's enamel antagonist, flexure strength, and surface roughness.

\section{Enamel wear}

Mulay., et al. "evaluated the wear of human enamel opposed by feldspathic leucite ceramics of different surface finishes. Four different surface finish methods were assessed: Auto-glazing, over glazing, polishing with the Shofu polishing kit (SHOFU Dental GmbH, Germany), polishing with DFS polishing wheels and paste. They concluded that porcelain should be polished instead of over-glazed, rationalizing that porcelain can inflict potential damage upon enamel. Enamel wear produced by polished porcelain is substantially less than auto-glazed and over-glazed porcelain. No significant difference was found in enamel wear when opposed by ceramic surfaces polished by two different methods [27].

Celtra Duo $^{\mathrm{TM}}$ (Dentsply, Degudent) zirconia reinforced lithium silicate has two post milling protocols. 1) Mill and cement, both of

Citation: Alaa Samir El-Ashkar and Omnia Nabil. "Recent Developments and Potential Challenges in Dealing with Internal and External Surfaces of Glass Matrix and Resin Matrix Ceramic Materials. A Literature Review". Acta Scientific Dental Sciences 6.3 (2022): 61-72. 
which require only polishing prior to cementation.2) mill, glaze, and cement, which necessitates the use of a glaze prior to cementation. D'Arcangelo., et al. "[28] studied the wear of glazed Celtra Duo and polished Celtra Duo in comparison to enamel. Both had similar values, but the glaze fired Celtra Duo showed less wear than the ground Celtra Duo. De Angelis., et al. "[29] recommended the glaze firing cycle as an important procedure that may improve the wear resistance of zirconia reinforced lithium silicate based ceramic.

\subsubsection{The effect of different finishing protocols on flexural strength}

Concerning the flexure strength, Mohammadibassir., et al. "study showed that the flexural strength of lithium disilicate ceramic after polishing with the OptraFine system (Ivoclar Vivadent AG) was similar to that after glazing. Owing to the finer diamond particles of the polishing paste, the system showed fewer surface flaws, cracks, porosities, and irregularities, which might be the reason for the increased flexural strength [30].

Mohammadibassir., et al. "reported that the three-step polishing kit with a polishing paste used for polishing of lithium disilicate (IPS e. max CAD) ceramics yields higher flexural strength than the two step kit. Despite similar surface roughness after polishing with the two systems. The author justified that it might be because of the extra step in the three-step process rather than the two-step method and the use of polishing paste with ultra-fine diamond paste. It is believed that application of diamond polishing paste decreases the surface roughness.30 Steiner., et al. "31 and Hanouf., et al. " [32] also reported that addition of a polishing step with diamond paste significantly improved surface smoothness of lithium disilicate allceramic systems. This may explain the lower surface roughness (both quality and quantity) in the three step + polish paste group in Mohammadibassir., et al. “[30].

The effect of different finishing protocols on surface roughness

Vichi., et al. "found that manual finishing/polishing of Lithium disilicate and zirconia reinforced lithium silicate using Optra fine kit (Ivoclar Vivadent AG) and Vita polishing kit (Vita Zahnfabrik, Germany) for 60 seconds, allows glass ceramics to yield a higher gloss similar to the primary glaze layer [33] These findings were in agreement with Lawson., et al. "who reported less efficacy of the glazing paste when compared with 60 -second manual polishing [34].
Regarding the material, zirconia reinforced lithium silicate (VITA Suprinity", VITA Zahnfabrik) displayed higher polishability than lithium disilicate (IPS e.max CAD). Vichi., et al. "justified that the finer microstructure and zirconia oxides of zirconia reinforced lithium silicate allow the material to be more efficaciously polished [33]. Incesu., et al. "studied the effects of different polishing systems on feldspathic and fluoroapatite ceramics and compared them to glazing. The OptraFine polishing kit and paste application can be used as an alternative to re-glazing since it achieves the smoothest surface in both groups, comparable to the glazed [35].

Vo., et al. "lithium disilicate (IPS e.max CAD) treated with glazing spray had the highest superficial roughness among the finishing systems. This might be due to the surface irregularities underneath the glaze layer. Glazing spray was not able to uniformly coat all the irregularities, thus resulting in a superficial roughness [36]. Fasbinder, et al. "recommended polishing prior to glazing, thus minimizing surface irregularities and obtaining a homogenous glaze coating [37]. For the glaze paste, Vichi., et al. "found that the paste resulted in a different glaze spread ability on the ceramic surface, which led to a variation of gloss and refractive index values [33].

Sgura., et al. "compared the effect of the Co2 laser on the glazing of feldspathic and leucite-based ceramics and compared it to autoglazing. Continuous CO2 Laser was as effective as conventional oven auto-glaze when applied to the porcelain surface.In conclusion, CO2 laser can be used as a chairside alternative to auto-glaze, because it is less time-consuming and similar results with regard to mechanical properties, $\mathrm{CO} 2$ laser glazing resulted in an increase in micro-hardness and fracture toughness of porcelain restorations. ${ }^{38}$ Regarding the colour parameters, $\mathrm{CO} 2$ laser was similar to auto-glaze in high surface gloss with no translucency or no colour changes [39].

\section{Resin matrix ceramics}

The 2013 edition of the American Dental Association Code on Dental Procedures and Nomenclature defines the term "porcelain" as "pressed, fired, polished, or milled materials containing predominantly inorganic refractory compounds, including porcelain, glass, ceramics, and glass-ceramics [40]. Resin-matrix ceramics consist of an organic matrix heavily filled with ceramic particles. They are composed predominantly ( $>50 \%$ by weight) of refractory inorganic compounds, irrespective of the presence of a less predominant organic phase (polymer) [40]. The inorganic composition of resin-

Citation: Alaa Samir El-Ashkar and Omnia Nabil. "Recent Developments and Potential Challenges in Dealing with Internal and External Surfaces of Glass Matrix and Resin Matrix Ceramic Materials. A Literature Review". Acta Scientific Dental Sciences 6.3 (2022): 61-72. 
matrix ceramics varies significantly; "Resin Nano Ceramic" as Lava Ultimate from $3 \mathrm{M}$ ESPE, is comprised of nanoceramic particles zirconia-silica nano-fillers in the form of dispersed or aggregated particles (79 percent by weight) and urethane dimethacrylate (UDMA) as the matrix [41]. VITA Enamic from VITA is a "Polymer Infiltrated Ceramic Network", a new type of interpenetrating phase material that has been developed (PICN). It has two interconnected threedimensional network structures: a polymer network reinforces the dominant fine-structure feldspar ceramic network (86 percent by weight or 75 percent by volume). ${ }^{41}$ The "Flexible Nano Ceramic" CERASMART $^{\text {TM }}$ from GC is made up of small, uniformly dispersed alumina-barium-silicate particles embedded in a polymer matrix $[40,41]$. For CAD/CAM restorations, resin matrix ceramics are specifically designed. In comparison to other ceramic materials, the milling time in the CAM unit is reduced, and the milling burs have a longer lifespan. After milling, there is no requirement for glazing or crystallization cycles; surface polishing can create the final shine and smoothness of the restoration [41].

Internal surface treatment protocols for resin matrix ceramics Chemical and mechanical surface treatment methods

It's approved that surface conditioning is essential for optimum bonding in resin matrix ceramics. Attributable to their variation in inorganic composition, the internal surface treatment methods differ from one class to another. Several methods, including sandblasting $50 \mathrm{um}$ of $\mathrm{Al} 2 \mathrm{O} 3$ at 2 bar for 5 seconds and $5 \% \mathrm{HF}$ for 60 seconds followed by silane application, have been used and mentioned in recent literature. Others recommended combining a universal adhesive containing MDP to increase the adhesive luting of Lava Ultimate and Vita Enamic [42]. Demirtag., et al. "HF was effective for Vita Enamic. After silanation, the Vita Enamic should have higher shear bond strength than that of sandblasted Vita Enamic. This is due to the fact that the material has a silica-containing feldspathic matrix [43].

\section{Laser surface treatment}

Akpinar., et al. "reported that a femtosecond laser produces homogeneous roughness without causing structural changes and thermal damage on the material surface by avoiding heat transfer on the surface using ultrashort laser pulses [13]. Demirtag., et al. "concluded that femtosecond laser irradiation was found to be more effective than sandblasting or HF acid etching for both Vita Enamic and Lava Ultimate in terms of surface roughness and bond strength to resin cement. There is scarce evidence regarding the effect of the femtosecond laser on crack propagation and the monomer chemistry [43]. In Barutcigil., et al. "study, the Er: YAG laser showed comparable bond strength values to those of sandblasting and cojet groups in the Vita Enamic surface treatment evaluation. While the HF etching reported the highest value [44]. Femtosecond laser, HF, and sandblasting surface treatments followed by silanization are recommended to condition the surface of Vita Enamic restoration $[43,44]$. Femtosecond laser and surface priming with silane coupling agent is recommended to condition the surface of Lava Ultimate restoration. Resin nanoceramics such as CERASMART are similar to Lava Ultimate. Surface roughening prior to silane application seemed essentially significant. HF acid caused a superficial dissolution of the ceramic silica components and an ineffective low surface roughness. Yet, they achieved high bond strength values. When compared to Lava Ultimate, HF etching of Lava Ultimate resulted in lower bond strength. Similarly, sandblasting Al203-blasting ( $50 \mu \mathrm{m} / 1$ bar) achieved similar bond strength values and strong roughness [9].

Different external surface treatments of resin matrix ceramics modalities and comparisons

\section{Using products' kit and customized polishing kits}

Sarikaya., et al. "evaluated the effects of different intraoral polishing kits on surface roughness of Vita Enamic, and Lava Ultimate material. Surface roughness values of Enamic specimens polished with Vita Enamic Polishing Kit (Vita Zahnfabrik, Germany), and Lava Ultimate specimens polished with Lava Meisinger Luster Polishing Kit (3M ESPE, MN, USA) have shown the lowest surface roughness values with no statistical difference from each other. For both ceramic types, there is no difference between Soflex (3M Espe, MN, USA) and shofu (custom kits) polished in terms of surface roughness. The authors'suggests that Soflex, and Shofu (Shofu Inc, Kyoto, Japan) may be used instead of the ceramics' own polishing kits [45].

\section{Low viscosity coating, compositeand ceramic polishing kits}

Sagsoz., et al. "evaluated the staining resistance of Lava Ultimate, CERASMART, Vita Enamic, polished with two different kits, Liquid polish used with compared to a glazed glass-ceramic. The staining resistance of Vita Enamic and CERASMART were significantly higher than that of Lava Ultimate. Both ceramic and composite polishing kits can be used for resin ceramics. Liquid polish has limited indications for resin ceramics [46]. 


\section{Wear behavior of material and enamel wear}

A 6-month clinical evaluation of the wear amount of CERASMART and Vita Enamic single molar crowns and opposing natural teeth using 3D image alignment. Both groups received the same polishing protocol (GC05 Twist Polisher Kit, Meisinger). For the Vita Enamic, which showed the highest enamel wear, the author deduced that the early abrasion of the resin parts led to the exposure of the ceramic network, which is more abrasive in nature than enamel. For the same reason, the CERASMART showed a faster rate of loss of their polished surface in the first 3 months, which led to a higher degree of self-wear. Based on the Aladağ., et al. "findings, the resin matrix ceramics should be polished every 3 months, contrary to the manufacturer's recommendations for intraoral re-polishing every 6 months [47].

\section{Intra-oral repair}

Resin ceramics have unique characteristics compared to ceramics. Their composition accepts addition and allows repairing intraorally. In general, grinding with water-cooled, medium grain diamond burs is one of the most common intra-oral repair protocols for resin matrix ceramics in clinical practice. Other methods such as, 5\% HF, Cojet, and sandblasting were also reviewed in the literature. These studies recommended silane application after surface conditioning with universal bonding agent followed by low viscosity composite [9]. Recently, the Nd: YAG laser proved superiority over the tribochemical Silica-coating and no treatment groups in bonding indirect composite veneer layers to Lava Ultimate, CERASMART, and Vita Enamic. As regards the materials, the Vita Enamic group found that the tribochemical silica coating technique significantly improved the shear bond strength compared to laser treatment. The study advises whether an Nd: YAG laser should be used, taking into consideration the microstructure and composition of resin matrix ceramic materials and appropriate parameters for each material.

\section{Conclusion}

One of the most essential factors of long-term clinical success is the correct bonding protocol between ceramics and resin cements. Recent research work into ceramic resin bonding has resulted in various new insights. Based on scientific and clinical evidence, it appears that glass ceramic restorations require HF etching and silanization as a chemical surface treatment method. However, the ideal way of bonding is still up for debate. Most of the research rec- ommended either HF etching or air particle abrasion based on the resin matrix ceramic microstructure, yet the number of available resin matrix ceramic and resin cement bonding studies is small. Silanization is still the most approved primer, and new monomers have shown promise in ceramic resin bonding. More lab investigations mimicking the oral environment are required for more reliable results. Clinical investigations on the effects of post-milling procedures such as polishing, glazing, and re-glazing are rare, and should be encouraged. However, the enormous number of variables such as differences in ceramic type, microstructure, and processing mode that influence the final outcome of exterior surface treatments should be considered. This review revealed the need for standardisation of methodologies, enabling comparisons between researches.

\section{Bibliography}

1. T Tian., et al. "Aspects of bonding between resin luting cements and glass ceramic materials". Dental Materials 30 (2014): e147-e162.

2. R Ramakrishnaiah., et al. "The Effect of Lithium Disilicate Ceramic Surface Neutralization on Wettability of Silane Coupling Agents and Adhesive Resin Cements". Silicon 10 (2018): 23912397.

3. GC Lopes., et al. "Does a Self-etching Ceramic Primer Improve Bonding to Lithium Disilicate Ceramics? Bond Strengths and FESEM Analyses". Operative Dentistry 44 (2019): 210-218.

4. D Sundfeld., et al. "The Effect of Hydrofluoric Acid Concentration and Heat on the Bonding to Lithium Disilicate Glass Ceramic". Brazilian Dental Journal 27 (2016): 727-733.

5. DM Mallikarjuna., et al. "Comparative evaluation of lithium disilicate ceramic surface and bond strength to dentin surface after treatment with hydrofluoric acid and acidulated phosphate fluoride gel: An In Vitro study". Indian Journal of Dental Research 29 (2018): 794-798.

6. E Volkel T and Braziulis. "Monobond etch and prime". Scientific Documentation (2015).

7. A Straface., et al. "HF etching of CAD/CAM materials: influence of HF concentration and etching time on shear bond strength". Head and Face Medicine 15 (2019): 21.

8. CO Uwalaka., et al. "Effect of sandblasting, etching and resin bonding on the flexural strength/bonding of novel glass-ceramics". Dental Materials 34 (2018): 1566-1577. 
9. T Strasser., et al. "Roughness, surface energy, and superficial damages of CAD/CAM materials after surface treatment". Clinical Oral Investigations 22 (2018): 2787-2797.

10. JP Matinlinna., et al. "Silane adhesion mechanism in dental applications and surface treatments: A review". Dental Materials 34 (2018): 13-28.

11. A Mirhashemi., et al. "Evaluation of Different Types of Lasers in Surface Conditioning of Porcelains: A Review Article". Journal of Lasers in Medical Science 8 (2017): 101-111.

12. L Liu., et al. "Effect of Nd: YAG laser irradiation on surface properties and bond strength of zirconia ceramics". Lasers in Medical Science 30 (2015): 627-634.

13. YZ Akpinar., et al. "Effect of Femtosecond Laser Treatment on the Shear Bond Strength of a Metal Bracket to Prepared Porcelain Surface". Photomedicine and Laser Surgery 33 (2015): 206-212.

14. T Sriamporn., et al. "Effect of Different Neutralizing Agents on Feldspathic Porcelain Etched by Hydrofluoric Acid". European Journal of Dentistry 13 (2019): 75-81.

15. A Aladağ., et al. "Effect of Different Cleaning Regimens on the Adhesion of Resin to Saliva-Contaminated Ceramics". Journal of Prosthodontics 24 (2015): 136-145.

16. F Yoshida., et al. "Influence of surface treatment of contaminated lithium disilicate and leucite glass ceramics on surface free energy and bond strength of universal adhesives". Dental Materials Journal 34 (2015): 855-862.

17. DM Dos Santos., et al. "Bond strength of lithium disilicate after cleaning methods of the remaining hydrofluoric acid". Journal of Clinical and Experimental Dentistry 12 (2020): e103-e107.

18. HJ Kim., et al. "Effects of silane treatment on salivary protein contamination during the bonding of lithium disilicate ceramic". Journal of Adhesion Science and Technology 34 (2020): 1885-1897.

19. B Lapinska., et al. "Effect of Surface Cleaning Regimen on Glass Ceramic Bond Strength”. Molecules 24.3 (2019): 389.

20. R Ramakrishnaiah., et al. "Two-step vs. one-step conditioning systems and adhesive interface of glass ceramic surface and resin systems". Journal of Adhesion Science and Technology 32 (2018): 1952-1963.
21. AFV Moro., et al. "Effect of prior silane application on the bond strength of a universal adhesive to a lithium disilicate ceramic". Journal of Prosthetic Dentistry 118 (2017): 666-671.

22. T Abduljabbar., et al. "Influence of silane and heated silane on the bond strength of lithium disilicate ceramics - An in vitro study". Journal of Pakistan Medical Association 32 (2016): 550554.

23. RF De Carvalho., et al. "Heat Treatment of Pre-hydrolyzed silane increases adhesion of phosphate monomer-based resin cement to glass ceramic". Brazilian Dental Journal 26 (2015): 44-49.

24. Y Maruo., et al. "Does 8-methacryloxyoctyl trimethoxy silane (8-MOTS) improve initial bond strength on lithium disilicate glass ceramic?" Dental Materials 33 (2017): e95-e100.

25. TA Hamza and RM Sherif. "Fracture Resistance of Monolithic Glass-Ceramics Versus Bilayered Zirconia-Based Restorations". Journal of Prosthodontics 28 (2019): e259-e264.

26. G Mulay., et al. "An evaluation of wear of human enamel opposed by ceramics of different surface finishes". The Journal of Indian Prosthodontic Society 15 (2015): 111-118.

27. CD'Arcangelo., et al. "Wear properties of dental ceramics and porcelains compared with human enamel". Journal of Prosthetic Dentistry 115 (2016): 350-355.

28. F De Angelis., et al. "Wear Properties of Different Additive Restorative Materials Used for Onlay/Overlay Posterior Restorations". Operative Dentistry 45 (2020): E156-E166.

29. M Mohammadibassir., et al. "Effect of Two Polishing Systems on Surface Roughness, Topography, and Flexural Strength of a Monolithic Lithium Disilicate Ceramic". Journal of Prosthodontics 28 (2019): e172-e180.

30. R Steiner., et al. "Adjusting dental ceramics: An in vitro evaluation of the ability of various ceramic polishing kits to mimic glazed dental ceramic surface". Journal of Prosthetic Dentistry 113 (2015): 616-622.

31. A Hanouf., et al. "Comparison of Surface Roughness of Ceramics after Polishing with Different Intraoral Polishing Systems using Profilometer and SEM". Journal of Dental Health, Oral Disorders and Therapy (2015). 
32. A Vichi RF., et al. "Effect of Finishing and Polishing on Roughness and Gloss of Lithium Disilicate and Lithium Silicate Zirconia Reinforced Glass Ceramic for CAD/CAM Systems". Operative Dentistry 43 (2018): 90-100.

33. Lawson S Janyavula., et al. "Wear of enamel opposing zirconia and lithium disilicate after adjustment, polishing and glazing". Journal of Dentistry 42 (2014): 1586-1591.

34. E Incesu and N Yanikoglu. "Evaluation of the effect of different polishing systems on the surface roughness of dental ceramics". Journal of Prosthetic Dentistry 124 (2020): 100-109.

35. DT Vo., et al. "Adherence of Streptococcus mutans on lithium disilicate porcelain specimens". Journal of Prosthetic Dentistry 114 (2015): 696-701.

36. DJ Fasbinder and GF Neiva. "Surface Evaluation of Polishing Techniques for New Resilient CAD/CAM Restorative Materials". Journal of Esthetic and Restorative Dentistry 28 (2016): 56-66.

37. R Sgura., et al. "Surface treatment of dental porcelain: CO2 laser as an alternative to oven glaze". Lasers in Medical Science 30 (2015): 661-667.

38. R Sgura., et al. "CO2 Laser Glazing Treatment of a Veneering Porcelain: Effects on Porosity, Translucency, and Mechanical Properties". Operative Dentistry 40 (2015): 247-254.

39. S Gracis., et al. "A New Classification System for All-Ceramic and Ceramic-like Restorative Materials". The International Journal of Prosthodontics 28 (2016): 227-235.

40. E Bajraktarova-Valjakova., et al. "Contemporary Dental Ceramic Materials, A Review: Chemical Composition, Physical and Mechanical Properties, Indications for Use". Open Access Macedonian Journal of Medical Sciences 6 (2018): 1742-1755.

41. MB Kömürcüoğlu., et al. "Influence of different surface treatments on bond strength of novel CAD/CAM restorative materials to resin cement". The Journal of Advanced Prosthodontics 9 (2017): 439-446.

42. Z Demirtag and AK Culhaoglu. "Surface Roughness of CeramicResin Composites After Femtosecond Laser Irradiation, Sandblasting or Acid Etching and Their Bond Strength with and Without Silanization to a Resin Cement". Operative Dentistry 44 (2019): 156-167.

43. K Barutcigil., et al. "Effect of Different Surface Treatments on Bond Strength of Resin Cement to a CAD/CAM Restorative Material". Journal of Prosthodontics 28 (2019): 71-78.
44. I Sarikaya and C Gulec. "The Effect of Polishing on Surface Roughness and Fracture Load Of Hybrid and Resin Nanoceramics". International Journal of Medical Dentistry 23 (2019).

45. O Sagsoz., et al. "The effects of different polishing techniques on the staining resistance of CAD/CAM resin-ceramics". The Journal of Advanced Prosthodontics 8 (2016): 417-422.

46. A Aladağ., et al. "In vivo wear determination of novel CAD/ CAM ceramic crowns by using 3D alignment". The Journal of Advanced Prosthodontics 11 (2019): 120-127.

47. E Çelik., et al. "Effect of surface treatments on the bond strength of indirect resin composite to resin matrix ceramics". The Journal of Advanced Prosthodontics 11 (2019): 223-231.

\section{Assets from publication with us}

- Prompt Acknowledgement after receiving the article

- Thorough Double blinded peer review

- Rapid Publication

- Issue of Publication Certificate

- High visibility of your Published work

Website: www.actascientific.com/

Submit Article: www.actascientific.com/submission.php Email us: editor@actascientific.com

Contact us: +919182824667 